\title{
Immune-inflammatory responses in the elderly: an update
}

\author{
Giulia Accardi ${ }^{1,2}$ and Calogero Caruso ${ }^{1,2^{*}}$
}

Keywords: Allergy, Cancer, Elderly, Immunosenescence, Immunestimulation, Inflammation, Vaccination

\section{Background}

In Western countries the number of persons 65 years or older is most rapidly increasing. The percentage of people over 60 in the world has risen from $9.2 \%$ in 1990 to $11.7 \%$ in 2013 and, according to several estimates, it will reach $21 \%$ by 2050 . It is expected to exceed the number of children for the first time in 2047. In addition, the number of persons above 80 years is projected to increase even more dramatically from a worldwide total of 125 million in 2015 to 434 million in 2050. Therefore, the problem of the elderly health is now a priority in medicine [1].

In May 2012, a group of scientists and clinicians developed a consensus statement to highlight the importance of a common view on ageing and healthy lifespan [2]. As reported in the panel, ageing is most likely one component of life, which first emerged in economically developed countries and results from a breakdown of self-organizing system and reduced ability to adapt to the environment. Ageing processes are defined as those that amplify the vulnerability of subjects, as they become older, to the factors that finally lead to death. In Western countries, the mortality rate increases 25 times more rapidly in individuals over 60 years old compared to people aged 25-44. Causes of death in aged are increased compared to individuals between 25 and 44 years old: cancer 43-fold, pneumonia and influenza 89-fold, heart diseases 92-fold, and stroke and chronic lung disease greater than 100 -fold. These data suggest a key role for immunity in the survival of the elderly because resistance to these diseases depends at least in part on optimal immune function [3].

\footnotetext{
* Correspondence: calogero.caruso@unipa.it

${ }^{1}$ Department of Pathobiology and Medical Biotechnologies, University of Palermo, Palermo, Italy

${ }^{2}$ Sezione di Patologia generale, Dipartimento di Biopatologia e Biotecnologie Mediche, Corso Tukory 211, 90134 Palermo, Italy
}

As reported in the Encyclopaedia of Immunobiology [4], in aged people, several changes of both innate and acquired immunity have been described and viewed as deleterious, hence the term immunosenescence. Thus, immunosenescence reflects age-related declines in immune function at the cellular and serological level. However, immunosenescence is a complex process involving multiple reorganizational and developmentally regulated changes, rather than simple unidirectional decline of complete immune function. On the other hand, some immunological parameters are commonly notably reduced in aged people. The principal immunosenescence hallmarks are represented by thymus involution with decreased new $\mathrm{T}$ cell generation and hematopoietic stem cell dysfunctions, decreased naïve and increased memory lymphocytes with accumulation of dysfunctional senescent cells with shortened telomeres. Defects in apoptotic cell death, mitochondrial function and stress responses, and malfunctioning of immune regulatory cells are also observed [5-7].

Immunosenescence is linked not only to the functional decline associated with the passage of time but also to antigen burden to which an individual has been exposed during lifetime. Therefore, a senescent immune system is characterized by continuous reshaping and shrinkage of the immune repertoire by persistent antigenic challenges. These changes lead to a poor response to newly encountered microbial antigens, including vaccines, as well as to a shift of the immune system towards an inflammatory, autoimmune, Th2 profile. This immune dysregulation provides the background for an increased susceptibility to autoimmune diseases, cancer, metabolic diseases, osteoporosis, neurological disorders, as well as to allergic inflammation and infections. Accordingly, the severity of many infections is higher in the elderly compared to younger adults and infectious diseases are frequently associated with long-term complications and 
frailty [4-8]. Reciprocally, an immune good function is tightly correlated to health status, as depicted in centenarian offspring $[9,10]$.

The chronic antigenic stress, which affects the immune system throughout life with a progressive activation of macrophages and related cells, contributes to determine an inflammatory status. The process of maintaining life for the individual is a constant struggle to preserve integrity. This can come at a price when immunity is involved, namely systemic inflammation. Inflammation is not per se a negative phenomenon. In fact, in response to cell injury elicited by trauma or infection the inflammatory response sets in, constituting a complex network of molecular and cellular interactions directed to facilitate a return to physiological homeostasis and tissue repair. If tissue health is not restored or the inflammatory trigger is not cleared, acute inflammation may become a chronic condition that continuously damages the surrounding tissues. The collateral damage caused by this type of inflammation usually accumulates slowly, sometimes asymptomatically for years but can eventually lead to severe tissue deterioration. Our immune system is, in fact, quite efficient in fighting acute infections in young people, but not particularly efficient in responding to chronic stimuli, especially when they occur late in life. This chronic state of low-grade inflammation, called inflamm-ageing, is characterized by increased levels of pro-inflammatory cytokines and acute phase proteins, which increase is a worse prognostic factor for all causes of death. This inflamm-ageing state is implicated in the pathogenesis of several age-related inflammatory diseases, such as atherosclerosis, diabetes, cancer and Alzheimer's disease $[11,12]$.

Inflamm-ageing status does not depend only on immunosenescence, but other factors play a role. One cause of inflamm-ageing is represented by damaged macromolecules and endogenous host-derived cell debris that accumulates over time and, as a consequence of their increased production and/or inadequate elimination, are sources of chronic tissue damage [13]. Cellular senescence consists in the irreversible cell cycle arrest generally due to telomeres shortening or cellular DNA damage and is central in the inflamm-ageing process. Moreover, senescent cells secrete various extracellular factors, including inflammatory cytokines and chemokines that can enhance and propagate senescence with autocrine and paracrine modality. Therefore, senescent cells contribute to the pro-inflammatory status of ageing [14]. Gut microbiota is a further player in the induction and maintenance of immunoregulatory circuits and tolerance. The alteration of gut microbiota composition, named dysbiosis, can determine immune dysregulation, and subsequent low-grade chronic inflammation also due to the systemic effect of bacteria lipopolysaccharides [15].
The increasing incidence of obesity represents a great challenge to global health. Along with cardiovascular disease and diabetes several studies also show an increased cancer risk in this population. Indeed, obesity is associated with an alteration of adipocytes functional activity that leads to a chronic and systemic inflammatory state. This inflammation is currently considered one of the main molecular links that foster cancer development in obese patients $[16,17]$.

However, the most relevant role is played by the dietary pattern. The Western-type diet, high in red meat, high-fat dairy products, refined grains and sugars, has been associated with higher levels of C-reactive protein (CRP) and interleukin(IL)-6. On the other hand, Mediterranean diet and more in general diets high in fruit and vegetable intake have been associated with lower levels of inflammation. Several researches have also associated specific nutrients with different level of inflammatory markers. The impact of different nutrients on the systemic inflammation has been experimentally condensed into one-dimensional numeric values. The "dietary inflammatory index" (DII) weights each major macronutrient and multiple micronutrients on the basis of their general pro-inflammatory effects, as measured, for example, by assessment of CRP in serum $[17,18]$.

Ageing is the major risk factor for cancer development. In the relationship between cancer and ageing, several factors play a role. Inflammation is the most relevant [19]. As previously stated, one hallmark of the ageing process is, in fact, represented by inflamm-ageing [20] and inflammation is also one hallmark of cancer [21]. In fact, chronic inflammatory diseases increase the risk of some cancers and strong epidemiological evidence exists that anti-inflammatory drugs are potent chemopreventive agents. The microenvironment of cancer contains many different inflammatory cells and mediators; the targeting of these factors in models decreases the development, growth and spread of cancer. Thus, inflammation offers targets both for the prevention and for cancer treatment. Thus, inflammaging represents the biological phenomena able to couple ageing process with cancer development [22, 23].

\section{The series}

To update some aspects of immune response in elderly, four reviews have been assembled in this series.

Leonardi et al., [18] have reviewed the molecular and cellular pathways involved in age-related chronic inflammation along with their potential triggers and their connection with cancer development. In particular, concerning diet and inflammation, they report that DII significantly correlates with an increased risk of postmenopausal breast cancer, colorectal cancer, lung cancer in smokers, nonHodgkin lymphoma, bladder cancer, and nasopharyngeal 
carcinoma. Given the evidence discussed above, it appears plausible to attempt dietary interventions also with food supplements to promote long-term systemic modulation of chronic low-grade inflammation process as an anticancer strategy and towards the improvement of health status in the elderly.

Di Lorenzo et al., [24] review the immunological and non-immunological mechanisms of allergic diseases in the elderly. In the last years the prevalence of allergic diseases in general population is increased because of environmental changes such as better hygiene, Westernized diet, air pollution, climate changes, and other factors that influence host microbiota. Additional factors are responsible for the increase of allergic diseases in elderly as the presence of several comorbidities that should interfere with the development and the type of allergic reactions. However, immunosenescence plays a central role by modifying response to microbiota and triggering inflamm-ageing. In addition, in elderly there is a shift from Th1 responses vs. Th2, hence favouring allergic responses.

The reviews of Weinberger [25] and Aspinall and Lang [26] discuss the prevention of infectious diseases from two different points of view. The Weinberger's paper regards vaccination as preventive measure against infections. Whilst vaccination is one of the most effective medical interventions ever introduced and has prevented millions of cases of infections worldwide every year, vaccines are often thought to be less effective in providing protection in this older section of society. One major reason for this statement is again the decline seen in effective immunity in this population. The analysis of the possible methods to reverse this decline is the topic of the second one. One approach has been to try to restore immunity within this population to something akin to that seen in younger individuals. Another has been to take a more practical approach believing that a weaker immune system may be provoked into providing a response if the stimulus is considerably strengthened and enhanced.

\section{Discussion}

Because ageing is an ineluctable process, strategies to live longer in a healthy condition have been the main goal of recent research. So, healthy ageing is the main challenge of the twenty-first century both in Western and developing countries [27].

In October 2013, a group of experts in biology and genetics of ageing developed a consensus related to the discovery and development of safe interventions to slow aging and increase healthy lifespan in humans [27]. As stated in the report of the workshop, accumulating scientific evidence from studies conducted in various models suggests that targeting ageing will not just postpone chronic diseases but also prevent multiple age-associated alterations while extending healthy lifespan. Interventions with the potential to target these pathways safely and to induce protective and rejuvenating responses that increase human health span are becoming available.

As suggested by studies conducted in model organisms, calorie restriction (CR), a reduction in food intake from $20 \%$ to $40 \%$, without malnutrition, extends maximum lifespan of various model organisms, including yeast, flies, worms and mammals [28].

Some studies in humans demonstrated that this effect seems to be linked to the quality of food rather than to quantity, so a dietary restriction (DR) rather than a CR, in particular to specific aminoacids, seems to be more effective. The result of these DRs is the down regulation of the so-called nutrient-sensing pathways: the insulin/ insulin growth factor(IGF)-1 and mTOR, and the reduction of IGF-1 level, differently from CR in which the level of IGF-1 is unchanged [29].

More interestingly, it was demonstrated that a periodic fasting mimicking diet (FMD) in mice (a very low calorie diet followed for five days) decreases inflammation and cancer incidence, other than neurodegeneration, extending lifespan in a good healthy status [30].

Similar experiment conducted in humans, although preliminary, demonstrated the reduction of some proposed markers of biological aging: CRP, systolic/diastolic blood pressure, and serum lipids. In addition, also IGF-1 and body mass index were reduced in these subjects. Thus, a sort of "rejuvenation" of human immune system and down regulation of ageing processes, after FMD, could be hypothesized, as demonstrated in mice who underwent to prolonged fasting (abstinence from foods but not water for two or more days) upon refeeding [31, 32].

A long life in a healthy, vigorous, youthful body has always been one of humanity's greatest dreams. In the Editorial and in the related papers, anti-ageing strategies have been discussed based on the control of immuneinflammatory responses, aimed not only to slow the ageing process, but also to delay or avoid the onset of age-related diseases such as cancer or combat infectious diseases. Intriguing there is a connection between these response and nutrient sensing pathways already known to delay age-related diseases and promote longevity [33].

\section{Abbreviations}

CR: Calorie restriction; CRP: C reactive protein; DIl: Dietary inflammatory index; DR: Dietary restriction; FMD: Fasting mimicking diet; IGF-1: Insulin growth factor-1; IL-6: Interleukin-6; mTor: Mammalian target of rapamycin

Acknowledgements

None. 


\section{Funding}

None.

\section{Availability of data and materials}

Not applicable.

\section{Provenance and peer review}

Commissioned; not externally peer reviewed.

Prof. Caruso is the Editor in Chief of Immunity \& Ageing, Dr. Accardi is member of the Editorial Board.

\section{Authors' contributions}

CC wrote the draft. Both authors edited the paper and approved its final version.

\section{Ethics approval and consent to participate}

Not applicable.

\section{Consent for publication}

Not applicable.

\section{Competing interests}

The authors declare they have no competing interests.

\section{Publisher's Note}

Springer Nature remains neutral with regard to jurisdictional claims in published maps and institutional affiliations.

Received: 31 December 2017 Accepted: 25 February 2018

Published online: 02 March 2018

\section{References}

1. Burch JB, Augustine AD, Frieden LA, Hadley E, Howcroft TK, Johnson R, Khalsa PS, Kohanski RA, Li XL, Macchiarini F, Niederehe G, Oh YS, Pawlyk AC, Rodriguez $\mathrm{H}$, Rowland $\mathrm{JH}$, Shen GL, Sierra F, Wise BC. Advances in geroscience: impact on healthspan and chronic disease. J Gerontol A Biol Sci Med Sci. 2014;69(Suppl 1):S1-3.

2. Avery P, Barzilai N, Benetos A, Bilianou H, Capri M, Caruso C, Franceschi C, Katsiki N, Mikhailidis DP, Panotopoulos G, Sikora E, Tzanetakou IP, Kolovou G. Ageing, longevity, exceptional longevity and related genetic and non genetics markers: panel statement. Curr Vasc Pharmacol. 2014;12:659-61.

3. Vasto $S$, Caruso C. Immunity \& Ageing: a new journal looking at ageing from an immunological point of view. Immun Ageing. 2004;1:1.

4. Caruso C, Vasto S. Immunity and aging. In: Ratcliffe MJH, editor. Encyclopedia of Immunobiology, Vol. 5, pp. Oxford: Academic Press; 2016. p. 127-32.

5. Wistuba-Hamprecht K, Haehnel K, Janssen N, Demuth I, Pawelec G. Peripheral blood T-cell signatures from high-resolution immune phenotyping of $\gamma \delta$ and $a \beta$ T-cells in younger and older subjects in the berlin aging study II. Immun Ageing. 2015;12:25.

6. Caruso C, Accardi G, Virruso C, Candore G. Sex, gender and immunosenescence: a key to understand the different lifespan between men and women? Immun Ageing. 2013;10:20.

7. Pawelec G. Hallmarks of human "immunosenescence": adaptation or dysregulation? Immun Ageing. 2012:9:15.

8. Caruso C, Candore G, Cigna D, DiLorenzo G, Sireci G, Dieli F, Salerno A. Cytokine production pathway in the elderly. Immunol Res. 1996;15:84-90.

9. Bucci L, Ostan R, Cevenini E, Pini E, Scurti M, Vitale G, Mari D, Caruso C, Sansoni P, Fanelli F, Pasquali R, Gueresi P, Franceschi C, Monti D. Centenarians' offspring as a model of healthy aging: a reappraisal of the data on Italian subjects and a comprehensive overview. Aging (Albany NY). 2016:8:510-9.

10. Pellicanò M, Buffa S, Goldeck D, Bulati M, Martorana A, Caruso C, ColonnaRomano G, Pawelec G. Evidence for less marked potential signs of T-cell immunosenescence in centenarian offspring than in the general agematched population. J Gerontol A Biol Sci Med Sci. 2014 May:69(5):495-504

11. Licastro F, Candore G, Lio D, Porcellini E, Colonna-Romano G, Franceschi C, Caruso C. Innate immunity and inflammation in ageing: a key for understanding age-related diseases. Immun Ageing. 2005;2:8.

12. Candore G, Caruso C, Jirillo E, Magrone T, Vasto S. Low grade inflammation as a common pathogenetic denominator in age-related diseases: novel drug targets for anti-ageing strategies and successful ageing achievement Curr Pharm Des. 2010;16:584-96.

13. Feldman N, Rotter-Maskowitz A, Okun E. DAMPs as mediators of sterile inflammation in aging- related pathologies. Ageing Res Rev. 2015;24:29-39.

14. Accardi G, Caruso C, Grillari J. From cellular senescence to age-associated diseases: miRNAs as tools and targets for healthy ageing. In: Accardi G, Caruso C, editors. Updates in pathobiology: causalityand chance in ageing, age-related diseases and longevity. Palermo: University Press; 2017. p. 27-37.

15. OToole PW, Jeffery IB. Gut microbiota and aging. Science. 2015;350:1214-5.

16. Balistreri CR, Caruso C, Candore G. The role of adipose tissue and adipokines in obesity-related inflammatory diseases. Mediat Inflamm. 2010;2010:802078.

17. Libra M, Nicoletti F. Diet, inflammation and cancer: a journey from prevention to treatment. In: Accardi G, Caruso C, editors. Updates in pathobiology: causality and chance in ageing, age-related diseases and longevity. Palermo: University Press; 2017. p. 145-62.

18. Leonardi GC, Accardi G, Monastero R, Nicoletti F, Libra M. Ageing: from inflammation to cancer. Immun Ageing. 2018;15:1

19. Vasto S, Carruba G, Lio D, Colonna-Romano G, Di Bona D, Candore G, Caruso C. Inflammation, ageing and cancer. Mech Ageing Dev. 2009;130:40-5.

20. López-Otín C, Blasco MA, Partridge L, Serrano M, Kroemer G. The hallmarks of aging. Cell. 2013;153:1194-217.

21. Hanahan D, Weinberg RA. Hallmarks of cancer: the next generation. Cell. 2011;144:646-74.

22. de Magalhães JP. How ageing processes influence cancer. Nat Rev Cancer. 2013;13:357-65.

23. Crusz SM, Balkwill FR. Inflammation and cancer: advances and new agents. Nat Rev Clin Oncol. 2015;12:584-96.

24. Di Lorenzo G, Di Bona D, Belluzzo F, Macchia L. Immunological and nonimmunological mechanisms of allergic diseases in the elderly: biological and clinical characteristics. Immun Ageing. 2017;14:23.

25. Weinberger B. Vaccines for the elderly: current use and future challenges. Immun Ageing. 2018;15:3

26. Aspinall $\mathrm{R}$, Lang $\mathrm{PO}$. Interventions to restore appropriate immune function in the elderly. Immun Ageing. 2018;15:5

27. Longo VD, Antebi A, Bartke A, Barzilai N, Brown-Borg HM, Caruso C, Curiel TJ, de Cabo R, Franceschi C, Gems D, Ingram DK, Johnson TE, Kennedy BK Kenyon C, Klein S, Kopchick JJ, Lepperdinger G, Madeo F, Mirisola MG, Mitchell JR, Passarino G, Rudolph KL, Sedivy JM, Shadel GS, Sinclair DA, Spindler SR, Suh Y, Vijg J, Vinciguerra M, Fontana L. Interventions to slow aging in humans: are we ready? Aging Cell. 2015;14:497-510.

28. Fontana L, Partridge L, Longo VD. Extending healthy life span-from yeast to humans. Science. 2010:328:321-6.

29. Fontana L, Weiss EP, Villareal DT, Klein S, Holloszy JO. Long-term effects of calorie or protein restriction on serum IGF-1 and IGFBP-3 concentration in humans. Aging Cell. 2008;7:681-7.

30. Brandhorst S, Choi IY, Wei M, Cheng CW, Sedrakyan S, Navarrete G, Dubeau L, Yap LP, Park R, Vinciguerra M, Di Biase S, Mirzaei H, Mirisola MG, Childress P, Ji L, Groshen S, Penna F, Odetti P, Perin L, Conti PS, Ikeno Y, Kennedy BK, Cohen $\mathrm{P}$, Morgan TE, Dorff TB, Longo VD. A periodic diet that mimics fasting promotes multi-system regeneration, enhanced cognitive performance, and Healthspan. Cell Metab. 2015;22:86-99.

31. Wei M, Brandhorst S, Shelehchi M, Mirzaei $H$, Cheng CW, Budniak J, Groshen S, Mack WJ, Guen E, Di Biase S, Cohen P, Morgan TE, Dorff T, Hong K, Michalsen A, Laviano A, Longo VD. Fasting-mimicking diet and markers/risk factors for aging, diabetes, cancer, and cardiovascular disease. Sci Transl Med. 2017;9(377)

32. Cheng CW, Adams GB, Perin L, Wei M, Zhou X, Lam BS, Da Sacco S, Mirisola M, Quinn DI, Dorff TB, Kopchick JJ, Longo VD. Prolonged fasting reduces IGF-1/PKA to promote hematopoietic-stem-cell-based regeneration and reverse immunosuppression. Cell Stem Cell. 2014;14:810-23.

33. Aiello A, Accardi G, Candore G, Gambino CM, Mirisola M, Taormina G, Virruso C, Caruso C. Nutrient sensing pathways as therapeutic targets for healthy ageing. Expert Opin Ther Targets. 2017:21:371-80. 